\title{
BMJ Open Mental health and well-being of older adults living with HIV in sub-Saharan Africa: a systematic review
}

\author{
Patrick Nzivo Mwangala (D) ,1,2 Adam Mabrouk, ${ }^{1}$ Ryan Wagner, ${ }^{3}$ \\ Charles R J C Newton, ${ }^{1,4}$ Amina A Abubakar ${ }^{1,4,5,6}$
}

To cite: Mwangala PN, Mabrouk A, Wagner R, et al. Mental health and well-being of older adults living with HIV in sub-Saharan Africa: a systematic review. BMJ Open 2021;11:e052810. doi:10.1136/ bmjopen-2021-052810

- Prepublication history and additional supplemental material for this paper are available online. To view these files, please visit the journal online (http://dx.doi.org/10.1136/ bmjopen-2021-052810).

Received 27 April 2021 Accepted 06 September 2021

\section{Check for updates}

(C) Author(s) (or their employer(s)) 2021. Re-use permitted under CC BY. Published by BMJ.

${ }^{1}$ Department of Clinical Research (Neurosciences), KEMRI-Wellcome Trust Research Programme, Kilifi, Kenya ${ }^{2}$ University of the Witwatersrand School of Public Health, Johannesburg, South Africa ${ }^{3} \mathrm{MRC} /$ Wits Rural Public Health and Health Transitions Research Unit (Agincourt), School of Public Health, Faculty of Health Sciences, University of the Witwatersrand, Parkton, Gauteng, South Africa

${ }^{4}$ Department of Psychiatry, University of Oxford, Oxford, UK ${ }^{5}$ Institute for Human Development, Aga Khan University, Nairobi, Kenya ${ }^{6}$ Department of Public Health, Pwani University, Kilifi, Kenya

Correspondence to Patrick Nzivo Mwangala; pmwangala27@gmail.com

\section{ABSTRACT}

Objective In this systematic review, we aimed to summarise the empirical evidence on common mental disorders (CMDs), cognitive impairment, frailty and healthrelated quality of life (HRQL) among people living with HIV aged $\geq 50$ years (PLWH50 +) residing in sub-Saharan Africa (SSA). Specifically, we document the prevalence and correlates of these outcomes.

Design, data sources and eligibility criteria The following online databases were systematically searched: PubMed, CINAHL, PsycINF0, Embase and Scopus up to January 2021. English-language publications on depression, anxiety, cognitive function, frailty and quality of life among PLWH50 + residing in SSA were included.

Data extraction and synthesis We extracted information, including study characteristics and main findings. These were tabulated, and a narrative synthesis approach was adopted, given the substantial heterogeneity among included studies.

Results A total of 50 studies from fifteen SSA countries met the inclusion criteria. About two-thirds of these studies emanated from Ethiopia, Uganda and South Africa. Studies regarding depression predominated $(n=26)$, followed by cognitive impairment $(n=13)$. Overall, PLWH50 + exhibited varying prevalence of depression (6\%-59\%), cognitive impairments $(4 \%-61 \%)$ and frailty $(3 \%-15 \%)$. The correlates of CMDs, cognitive impairment, frailty and $\mathrm{HRQOL}$ were rarely investigated, but those reported were sociodemographic variables, many of which were inconsistent.

Conclusions This review documented an increasing number of published studies on HIV and ageing from SSA. However, the current evidence on the mental and well-being outcomes in PLWH50 + is inadequate to characterise the public health dimension of these impairments in SSA, because of heterogeneous findings, few well-designed studies and substantial methodological limitations in many of the available studies. Future work should have sufficiently large samples of PLWH50+, engage appropriate comparison groups, harmonise the measurement of these outcomes using a standardised methodology to generate more robust prevalence estimates and confirm predictors.

PROSPERO registration number CRD42020145791.

\section{BACKGROUND}

All societies globally are experiencing an ageing population-some are in its early stages, and some are more advanced. ${ }^{1}$

\section{STRENGTHS AND LIMITATIONS OF THIS STUDY}

$\Rightarrow$ This is the first comprehensive review of common mental disorders, cognitive impairment, frailty and quality of life among older adults living with HIV in sub-Saharan Africa, synthesising the current evidence regarding the prevalence and correlates of these outcomes.

$\Rightarrow$ Our expanded search of five online databases and snowballing techniques minimised the risk of missing relevant studies.

$\Rightarrow$ Meta-analysis was not conducted due to extensive heterogeneity across the studies in methods and presentation of results.

$\Rightarrow$ We observed substantial methodological limitations in many of the included studies, for example, small sample sizes, making it difficult to characterise the real public health dimension of these impairments in the region.

Likewise, the proportion of people living with HIV (PLWH) aged $\geq 50$ years (considered as older adults within the HIV literature) is growing rapidly across the globe, ${ }^{2}$ demanding an increase in research, policy and practice to address the complex needs of this vulnerable group. ${ }^{3}$ In 2016, there were around 6 million PLWH50 + globally, representing $16 \%$ of the entire adult HIV population. ${ }^{2}$ This proportion was projected to reach $21 \%$ by the end of 2020, with Southern and Eastern Africa containing the vast majority of PLWH50+. ${ }^{2}$ The increase in older PLWH is mainly driven by two factors: effective antiretroviral therapy (ART) and new HIV infections among the elderly. ${ }^{4}$

This rapidly growing segment of PLWH50 + yields new challenges. Several HIV and ageing cohorts (mostly from highincome countries, HICs) are pointing out an elevated burden of physical conditions (eg, cardiometabolic diseases), mental morbidities (eg, depression, cognitive disorders), psychosocial challenges (eg, stigma, loneliness) and geriatric syndromes (eg, frailty) 
among PLWH $\geq 50$ years (PLWH50+) compared with their uninfected counterparts. ${ }^{4-6}$ Unfortunately, the mechanisms for the heightened risk of poor outcomes among PLWH50 + are not fully understood but span a host of disease-related factors, legacy of the early years of the HIV epidemic and host vulnerability factors. ${ }^{4}$ Frailty, common mental disorders (CMDs) such as depression and anxiety, and cognitive impairments are of particular concern in this population. ${ }^{4-6}$ Frailty is an important emerging indicator of vulnerability that is increasingly being evaluated among individuals ageing with HIV. ${ }^{7}$ It describes a state of physiological vulnerability that, in the presence of internal or external stressors, puts a person at an elevated risk of adverse clinical outcomes, such as hospitalisation, functional disability and mortality. ${ }^{89}$ Overall, mental morbidities and frailty are understudied in the literature despite their elevated burden and potential adverse impacts on HIV treatment and health-related quality of life (HRQoL).

Despite the apparent need to sharpen the focus on the needs of the growing cohort of PLWH50+, this population is being neglected in HIV care and prevention efforts, especially in low-income and middle-income settings of sub-Saharan Africa (SSA). In SSA, this concern is not just based on the close association between ageing and physical, cognitive, mental impairments, but also on the recognition that older adults are not considered to be a priority for policy issues. They are marginalised and increasingly vulnerable to greater poverty. ${ }^{1011}$ Thus, the burden and determinants of physical and mental impairments in these adults in SSA, the region most affected by HIV globally, are largely undocumented. ${ }^{12}$

The course and implication of physical and mental morbidities among PLWH50 + are well described in HICs, but it is an emergent research area in SSA. However, since 2010, the SSA region has witnessed a rise in interest in HIV and ageing outcomes, evidenced by the establishment of cohort studies of older people in different parts of Africa. ${ }^{13-15}$ This is encouraging since data from HICs may not be readily generalised to SSA where differences in, for example, genetics, social environmental milieu, formal and informal support systems will almost certainly alter the risk profile and morbidity characteristics among PLWH50+. Therefore, the current review aims to summarise the existing empirical evidence on CMDs, cognitive impairment, frailty and HRQoL among PLWH50 + in SSA by addressing the following specific objectives.

1. Establish the prevalence of CMDs, cognitive impairment and frailty among PLWH50 + in SSA.

2. Identify the correlates of CMDs, cognitive impairment, frailty and HRQoL among PLWH50 + in SSA.

\section{METHODS}

We followed the Preferred Reporting Items for Systematic Reviews and Meta-analyses guidelines to report the study. ${ }^{16}$

\section{Search strategy}

Five online databases (PubMed, CINAHL, PsycINFO, Embase and Scopus) were searched for publications. The last search was conducted on 4 January 2021. The following search terms were used: (HIV OR HIV-1 OR HIV/AIDS OR HIV infections) AND (adult OR older adult OR older people OR older individual OR elderly) AND (Africa OR sub-Saharan Africa OR Africa South of the Sahara) AND (cognitive impairment OR neurocognitive impairment OR neurological complication OR HIVassociated neurocognitive disorder OR common mental disorder OR depression OR depressive symptoms OR depressive disorder $\mathrm{OR}$ anxiety $\mathrm{OR}$ anxiety disorder $\mathrm{OR}$ quality of life OR health-related quality of life OR grip strength OR hand strength OR frailty). We also searched through the reference lists of included articles to access additional articles. Details of the full search strategy are provided in online supplemental file 1.

Studies were considered eligible if they: (1) involved PLWH50 + or had participants whose mean/median age was $\geq 50$ years or aggregated their outcomes of interest by age (including the $\geq 50$ age category); (2) were conducted within SSA; (3) were empirical and published in a peerreviewed journal and (4) reported any of our outcomes of interest (CMDs, cognitive impairment, frailty or HRQoL). We excluded studies that did not aggregate the outcomes of interest by HIV status. We also excluded studies that were published in non-English languages. Two of the authors (PNM and AM) independently screened the titles, abstracts and full articles for eligibility and reached consensus.

\section{Data extraction and quality assessment}

The two authors (PNM and AM) independently extracted the following study characteristics using a similarly designed extraction sheet: (1) first author; (2) country and the year of publication; (3) sample description; (4) how the outcome of interest was measured; (5) results reported; (6) key findings on the outcome of interest. For studies exclusively carried out among PLWH50+, we computed or extracted the reported percentages of the relevant outcomes. Similarly, for studies with middle-aged participants but whose mean/median age was $\geq 50$ years, we also computed or extracted the reported percentages for the relevant outcomes for the whole sample. For the studies with middle-aged participants (but whose mean/ median age was not at least 50 years), we computed percentages for participants who fell within the $\geq 50$ category, which was usually provided in the papers. In some studies, it was impossible to calculate these percentages. Hence, the occurrence of a specific outcome was reported in the original effect measure, for example, OR, median or mean. Any disagreement between the reviewers during data abstraction was also resolved by consensus.

We evaluated the quality of included studies using the modified version of the Newcastle-Ottawa Scale. ${ }^{17}$ The scale uses five parameters (sample size, sample representativeness, comparability between respondents and 
non-respondents, ascertainment of the outcome of interest, and quality of descriptive statistics reporting) to obtain a total score ranging from 0 to 5 . A score $\geq 3$ is considered a low risk of bias, while a score of $<3$ is regarded as a high risk of bias.

\section{Data synthesis and analysis}

A narrative synthesis approach was used to compare studies and describe the state of evidence on all reported outcomes; by describing the effect estimates, for instance, proportions, means with SD, and median with their IQRs. Where applicable, ranges were used to summarise the outcomes of interest reported. A meta-analysis could not be done due to a limited number of studies and substantial heterogeneity among studies.

\section{Patient and public involvement statement}

The accompanying manuscript is part of broader doctoral research work for the first author. During the conceptual phase of this work, community engagement through meetings with different stakeholders was carried out to contextualise the issues of ageing and HIV at the coast of Kenya (eg, in terms of priorities and preferences). These stakeholders included the Kilifi and Mombasa counties' departments of Health, subcounty health management teams, and hospital management teams of various health facilities at the study setting. The research team received and incorporated useful feedback from these meetings towards the design and implementation of the project. It also led to further elaborations regarding ongoing community engagement during and after the study, including disseminating any work arising from this project using these forums and others such as publications, hospital departmental meetings, patient groups and conferences as an essential way to discuss the implications of the findings. For this systematic review, patients were not involved in the conduct of the study. However, the results will be disseminated to the relevant patient groups within the HIV clinics through feedback meetings.

\section{RESULTS}

\section{Characteristics of included studies}

We identified 8741 articles from the 5 online databases and an additional 7 citations from snowballing. Of these, 2693 were duplicates. Hence, we screened 6055 titles and abstracts for initial eligibility, out of which 575 articles were identified. Full articles were obtained for these citations, of which 50 met the eligibility criteria (figure 1). The eligible studies were conducted between 2006 and 2021 among 15 SSA countries of Uganda $(n=13)$, South Africa $(n=12)$, Ethiopia $(n=8)$, Tanzania $(n=5)$, Nigeria $(n=3)$, Cameroon $(n=2)$, Côte d'Ivoire $(n=2)$, Senegal $(n=2)$, Kenya $(n=2)$, Botswana $(n=1)$, Zambia $(n=1)$, Malawi $(n=1)$, Namibia $(n=1)$, Togo $(n=1)$ and Burkina Faso $(n=1)$. However, some of these studies were conducted in multiple countries. Figure 2 shows the geographical distribution of these studies across the SSA region. Virtually

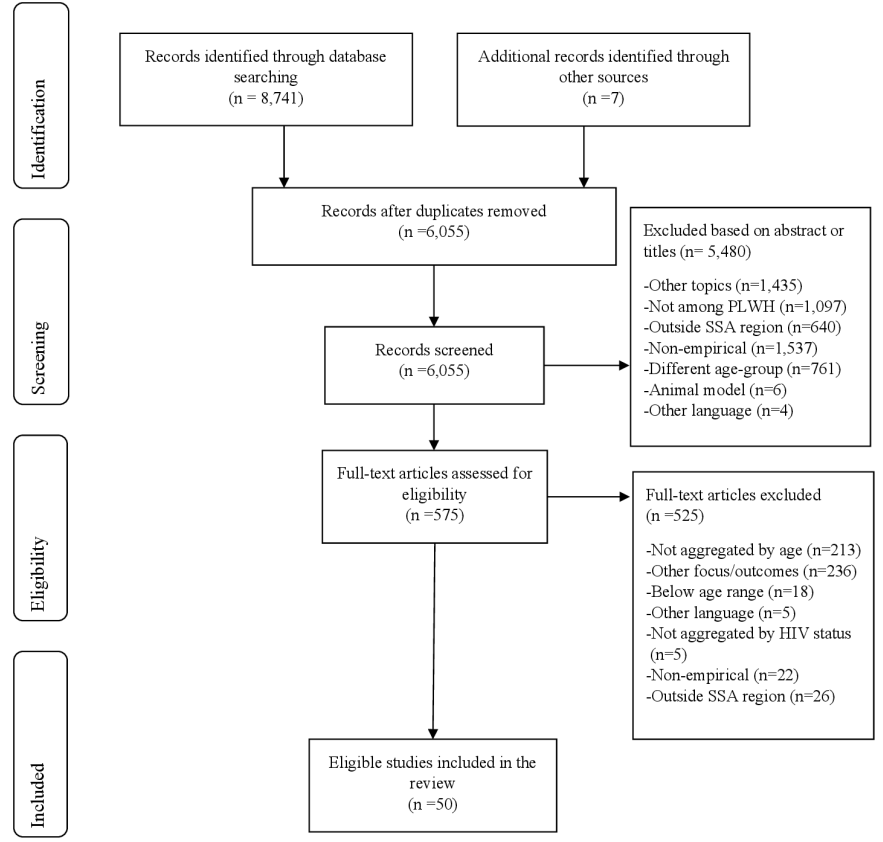

Figure 1 Identification of eligible studies. PLWH, people living with HIV; SSA, sub-Saharan Africa.

all the included studies were cross-sectional, except one that presented data from a randomised trial. ${ }^{18}$ Eighteen studies $(36 \%)$ analysed baseline or follow-up data of different cohort studies.

About two-thirds of the included studies $(n=35)$ recruited their HIV infected samples from clinical settings. ${ }^{18-52}$ The rest obtained their samples from the general population through household surveys and community samples. Additionally, many of the studies included samples of PLWH50 + lower than $100 \quad(n=22)$. Overall, the samples of PLWH50 + per study ranged from 19 to $1048,{ }^{36} 53$ while those for HIV uninfected older adults ranged from 17 to $4022 .^{22} 54$

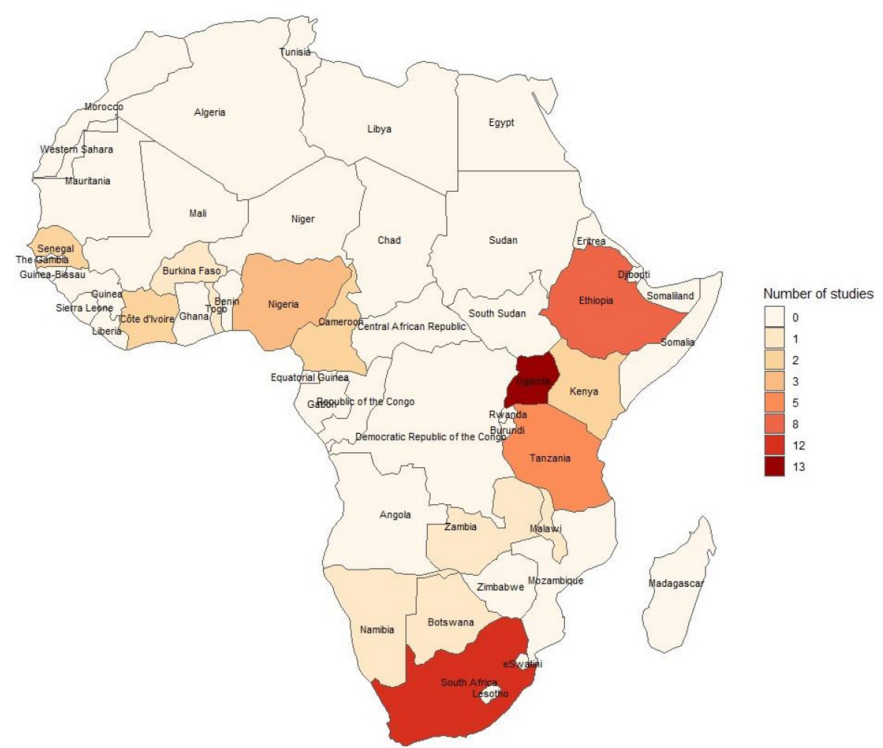

Figure 2 Geographical distribution of the included studies within SSA. SSA, sub-Saharan Africa. 
Common HIV disease markers such as the duration of HIV infection, current/nadir CD4 $+\mathrm{T}$ cell count, duration of ART use, viral suppression level among PLWH50 + were reported in a few studies. However, there was extensive heterogeneity in how the information was reported and the level of detail given. In $42 \%$ of the included studies, all PLWH50 + were on ART. Six other studies included only ART naïve PLWH50+. Among ART users, viral suppression was reported in five studies where it was documented to range from $73 \%$ to $90 \%$. Nadir CD4 +T cell count among PLWH50 + was reported in four studies where most of the participants (about $60 \%$ ) had a count lower than 200. The duration of HIV infection among PLWH50 + ranged from 3.5 to 12 years (among the few studies that reported this variable).

Only eight studies examined the health outcomes of interest using an exclusively HIV infected older sample. ${ }^{25} 334045-49$ Eighteen other studies used HIV uninfected older adults as comparison groups, while the rest included HIV infected young adults (the ages varied extensively).

\section{Depression and anxiety}

Depression was reported in 25 studies, while anxiety was reported in two studies. Notably, depression was assessed using multiple scales with diverse scoring systems. Six of the studies used the 9-item Patient Health Questionnaire (PHQ-9), while a similar number used the Centre for Epidemiological Studies Depression scale. Five other studies used the Mini International Neuropsychiatric Interview (MINI), while the rest utilised a host of different scales. On the other hand, anxiety was assessed using the Schedule for Clinical Assessment in Psychiatry and from clinical records. ${ }^{30}$ Nine of the depression studies $^{19} 2944495155-58$ reported major depressive disorders (MDD), while the remaining sixteen studies reported the prevalence of depressive symptoms. To synthesise and report the available evidence, depression (whenever used in this review) captures both the depressive symptoms (derived from brief screening measures) and Major Depressive Disorder (based on structured diagnostic interviews, for example, the MINI by specialised professionals or trained non-specialised workers). More information on this outcome is presented in the online supplemental table 1.

\section{Prevalence of depression and anxiety}

Overall, the prevalence of depression ranged from $6 \%$ to $59 \%$. We found no clear pattern of over-reporting or under-reporting of depression prevalence in the included studies, considering the different cut-offs used. For instance, in studies using the PHQ-9, the prevalence of depression ranged from $47 \%$ to $49 \%$ (with a cut-off of $\geq 5$ ) and $16 \%$ to $45 \%$ when using a cut-off of $\geq 10$. We also noted a similar observation when we took the ART status of PLWH into account. Specifically, the prevalence of depression ranged from $8 \%$ to $59 \%$ among studies whose participants were entirely on ART compared with
$6 \%$ to $54 \%$ among studies with mixed or naïve PLWH. However, studies that used structured interviews such as the MINI, Composite International Diagnostic Interview (CIDI) and Diagnostic and Statistical Manual of Mental Disorders, fourth edition (DSM-IV) to assess for depression were noted to frequently report lower prevalence of depression among PLWH50+ (ranging from 6\% to $30 \%)^{4457}$ compared with studies that used brief screening scales such as the PHQ-9 (prevalence ranging from $8 \%$ to $59 \%){ }^{2759}$

Of note, eight studies documented higher prevalence of depression among PLWH50 + than their comparison groups. ${ }^{2023273637435556}$ On the contrary, 11 studies reported a lower prevalence of depression among PLWH50 + than their comparison groups. ${ }^{19} 24282931324450515859$ In comparison, three other studies reported minimal or no differences in depression between PLWH50 + and their comparison groups. ${ }^{34257}$ In one population-based study conducted in rural South Africa, neither diagnosis nor treatment of HIV was significantly associated with depression when compared with being uninfected. ${ }^{54}$

Anxiety was reported in two studies. ${ }^{3039}$ In one of them, ${ }^{39}$ PLWH50 + on ART presented with the same proportion of anxiety symptoms with HIV uninfected older adults at $3 \%$. In the other study, ${ }^{30}$ the prevalence of anxiety disorders among PLWH50 + on ART was 21\% compared with $22 \%$ in HIV infected young adults (18-50 years).

\section{Reported correlates of depression}

Correlates of depression among PLWH50 + were reported only in eight studies. ${ }^{27} 28464851545558$ Among sociodemographic variables, older age, ${ }^{27} 54$ declining socioeconomic status, ${ }^{55}$ being unemployed, ${ }^{46}$ being female, ${ }^{28}{ }^{58}$ HIV status disclosure ${ }^{48}$ and urban residency ${ }^{58}$ were associated with an elevated risk of depression while being married, employed (full-time or part-time work),$^{54}$ old age, ${ }^{51}$ higher educational attainment ${ }^{48}$ and belonging to the highest wealth quintile ${ }^{28}$ were associated with reduced levels of depression. However, in some studies, household wealth quintile, education levels, ${ }^{51}$ and age ${ }^{25}$ were not significant correlates of depression. In other studies, being a current/former smoker, ${ }^{46}$ increasing disability scores, decreasing mean handgrip strength, reported back pain, not having hypertension, ${ }^{55}$ HIV stigma ${ }^{48}$ and caregiving (for adult children) ${ }^{58}$ were significantly associated with elevated levels of depression. On the other hand, receiving a government grant ${ }^{58}$ and resilience ${ }^{48}$ were significantly associated with reduced levels of depression. Details of this data are presented in online supplemental table 1.

\section{Cognitive and neurological functioning}

These outcomes were reported by 13 studies, as highlighted in table 1 . Of these, five studies quantified the prevalence of HIV-associated neurocognitive disorders (HAND) using different measures, ${ }^{25} 35384049$ two studies reported findings on dementia ${ }^{21}{ }^{60}$ while five studies documented findings on cognitive function/ 


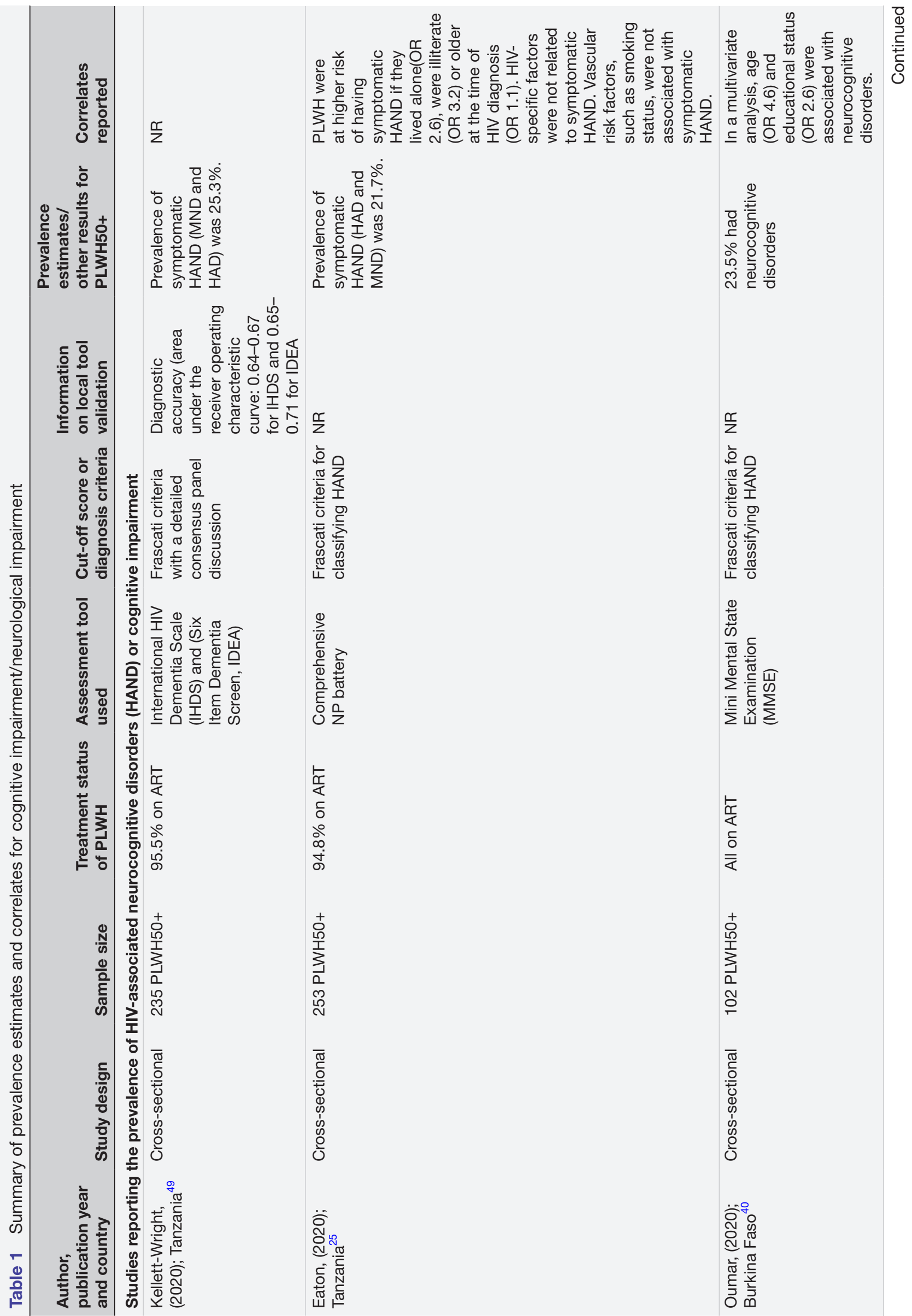




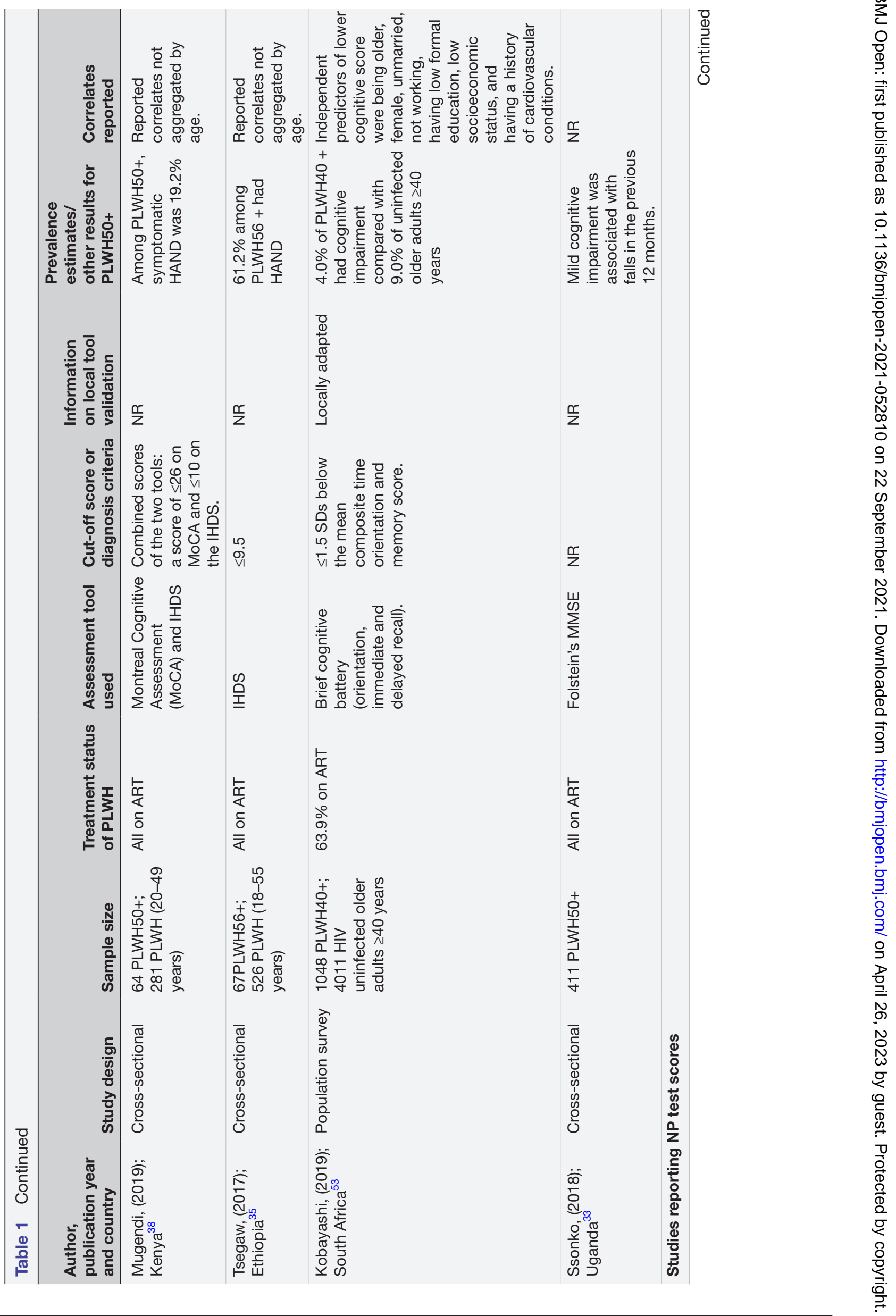




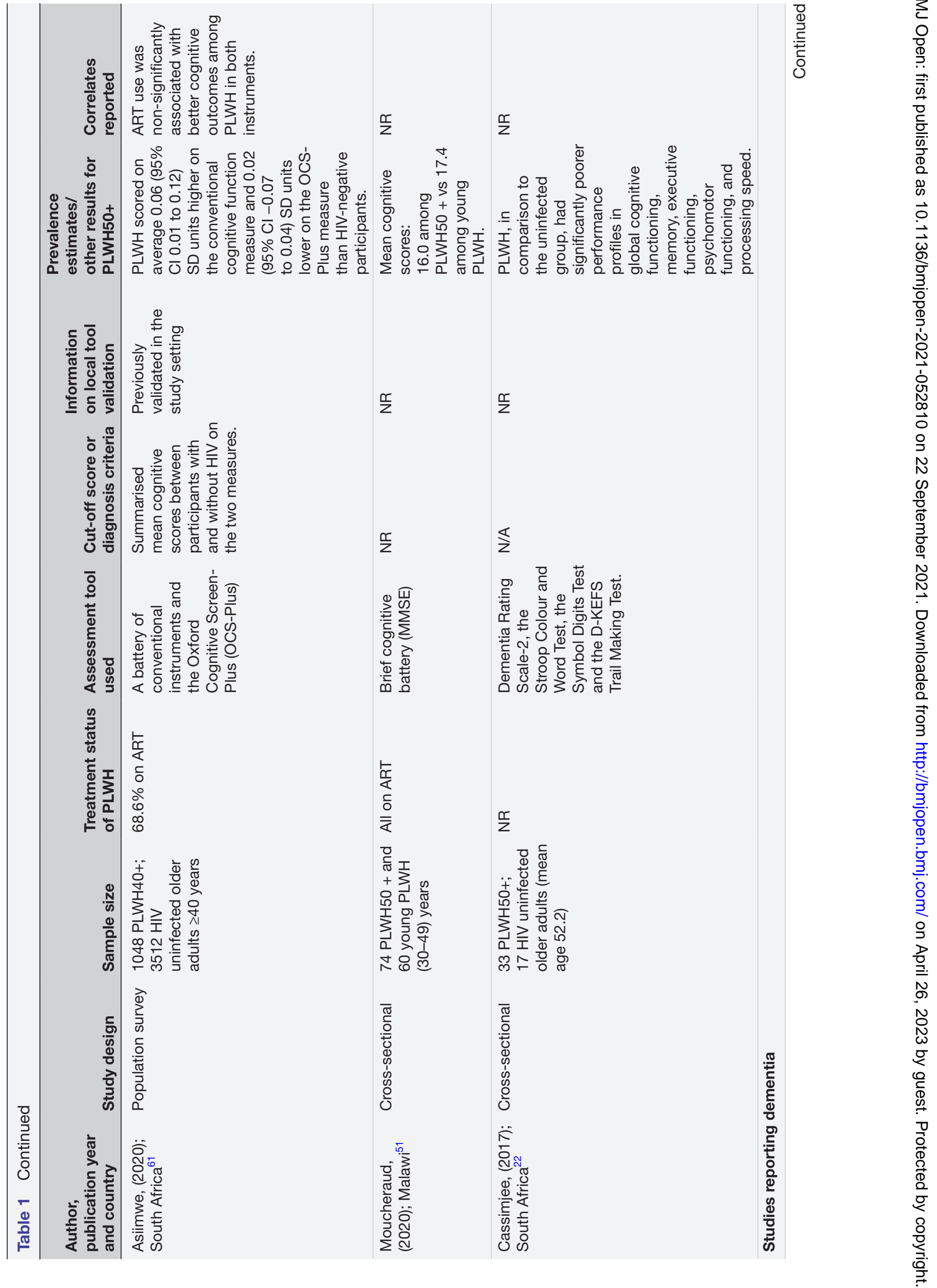




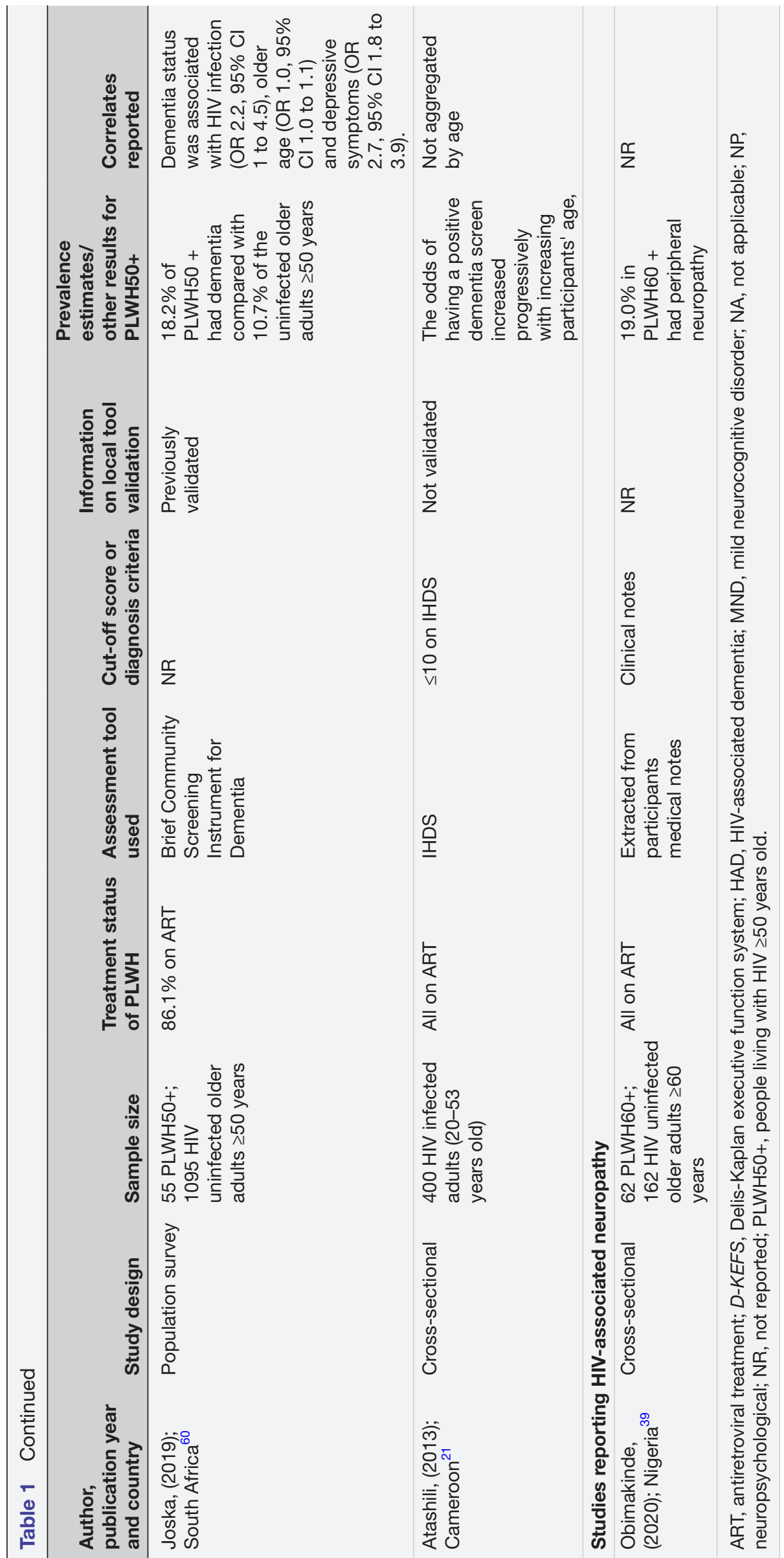


impairment. ${ }^{22} 33515361$ The remaining study reported the proportion of HIV-associated peripheral neuropathy among PLWH50+. ${ }^{39}$

\section{Prevalence estimates of reported outcomes}

The prevalence of symptomatic HAND among PLWH50 + on ART ranged from $19 \%{ }^{38}$ to $61 \% .{ }^{35}$ Two of the HAND studies documented elevated proportions of symptomatic HAND among PLWH50 + on ART compared with their younger counterparts. ${ }^{35}{ }^{38}$ In rural South Africa, Joska et al examined the prevalence of dementia and its associations in a population survey of 1150 rural elderly participants. They found no significant differences in the prevalence of dementia between PLWH50+ (86\% on ART) and HIV uninfected counterparts $18 \%$ vs $11 \%$, respectively. ${ }^{60}$ In a different study in rural South Africa, a baseline analysis of the Health and Ageing in Africa-A Longitudinal Study of an INDEPTH Community (HAALSI), HIV infected older adults ( $64 \%$ on ART) registered a lower prevalence of cognitive impairment (4\%) than their uninfected peers $(9 \%) .{ }^{53}$ In a separate analysis of the same baseline data of the HAALSI cohort, ${ }^{61}$ HIV infected older adults scored on average 0.06 (95\% CI 0.01 to 0.12 ) SD units higher on a conventional cognitive function measure and $0.02(95 \%$ CI -0.07 to 0.04$)$ SD units lower when using the Oxford Cognitive Screen-Plus (OCS-Plus) measure than their HIV-negative counterparts.

\section{Correlates of cognitive function/impairment}

The correlates of cognitive function/impairment were reported in seven studies. The key predictors of reduced cognitive function were mainly sociodemographic: older age ( $\geq 50$ years), ${ }^{21} 405360$ living alone, ${ }^{25}$ being illiterate or having low formal education level, ${ }^{25} 53$ being older at the time of HIV diagnosis, ${ }^{25}$ being female, unmarried and having low socioeconomic status ${ }^{53}$ were associated with a higher risk of impairment. However, in some studies, the effects of age, education and marital status were not significant. $^{25}{ }^{60}$ Other correlates of cognitive impairment included the presence of depressive symptoms, ${ }^{60} \mathrm{a}$ history of cardiovascular conditions ${ }^{53}$ and a history of falls in the past 12 months. ${ }^{33}$ HIV specific factors (such as nadir CD4 count, ART use) ${ }^{2561}$ and vascular risk factors (such as smoking status, body mass index and blood pressure $)^{25}$ were not associated with cognitive impairment.

\section{Frailty/grip strength}

\section{Prevalence estimates}

Frailty was reported in three studies. ${ }^{33} 4762$ In the first study, a hospital-based cross-sectional study of 145 PLWH50 + on ART in Tanzania, frailty assessment was completed using three measures (the Fried frailty phenotype (FFP), the Clinical Frailty Scale (CFS) and Brief Frailty Instrument for Tanzania (B-FIT 2)) ${ }^{47}$ In this study, the authors reported low levels of frailty: $3 \%$ (when using the FFP method) and 1\% (when using the CFS and BFIT-2 methods). The second study documented a similar prevalence of frailty among 292 PLWH50+ (ART status not reported) and 322 HIV uninfected peers in rural South Africa (15\% vs $18 \%$, respectively), using a modified version of the FFP. ${ }^{62}$ In the third study, increasing frailty scores were significantly associated with increasing polypharmacy levels in a sample of 411 PLWH50 + on ART in Uganda. ${ }^{33}$ Five other studies ${ }^{1845} 576364$ reported mean handgrip strength as an indicator of frailty, as shown in table 2.

\section{Reported correlates of frailty}

The correlates of frailty were reported in two studies. Being female, unmarried ${ }^{47}$ and older ${ }^{47} 62$ were significantly associated with worse frailty scores, and there were no associations between inflammatory biomarkers with frailty. ${ }^{62}$ Factors associated with mean handgrip strength were reported in four of the studies. In one, HIV status was significantly associated with weaker handgrip strength. ${ }^{57}$ In another study, being female and divorced/ widowed were significantly associated with weaker handgrip strength, while having a secondary education level was significantly associated with better handgrip strength. Table 2 gives details of this outcome.

\section{Health-related quality of life}

This outcome was reported in eight studies. Threequarters of the studies used the WHO Quality of Life measure to assess HRQOL. The overall mean quality of life scores (out of 100) ranged from 42 to 83 among PLWH50 + on ART. In two studies, PLWH50+ (partially on ART) reported better quality of life scores than their uninfected counterparts. ${ }^{63} 65$ In contrast, four other studies reported a lower quality of life scores ${ }^{39} 415266$ among PLWH50 + compared with to HIV uninfected older adults or HIV infected young adults.

The correlates of quality of life were reported in only one study, ${ }^{65}$ where having some source of income and being in the upper two wealth quintiles were significantly associated with better quality of life among PLWH50+. Additionally, being female and previously married were associated with reduced odds of good quality of life in PLWH50+. Table 3 gives more details on this outcome.

Overall, a significant number of the included studies (about $35 \%$ ) had a low quality score $(\leq 2-$ indicating a high risk of bias). ${ }^{19-22} 26293135-41485166$ Specifically, about two-thirds of the included studies did not report participant response rates. Sixteen studies used non-probability sampling methods or did not report their sampling methods. Close to half of the included studies had substantially small samples of PLWH50+ $(\leq 100)$. Furthermore, a substantial number of studies (about $40 \%$ ) provided scarce information regarding their measures' reliability and validity. Details of the quality assessment of all included studies are captured in online supplemental table 2. 


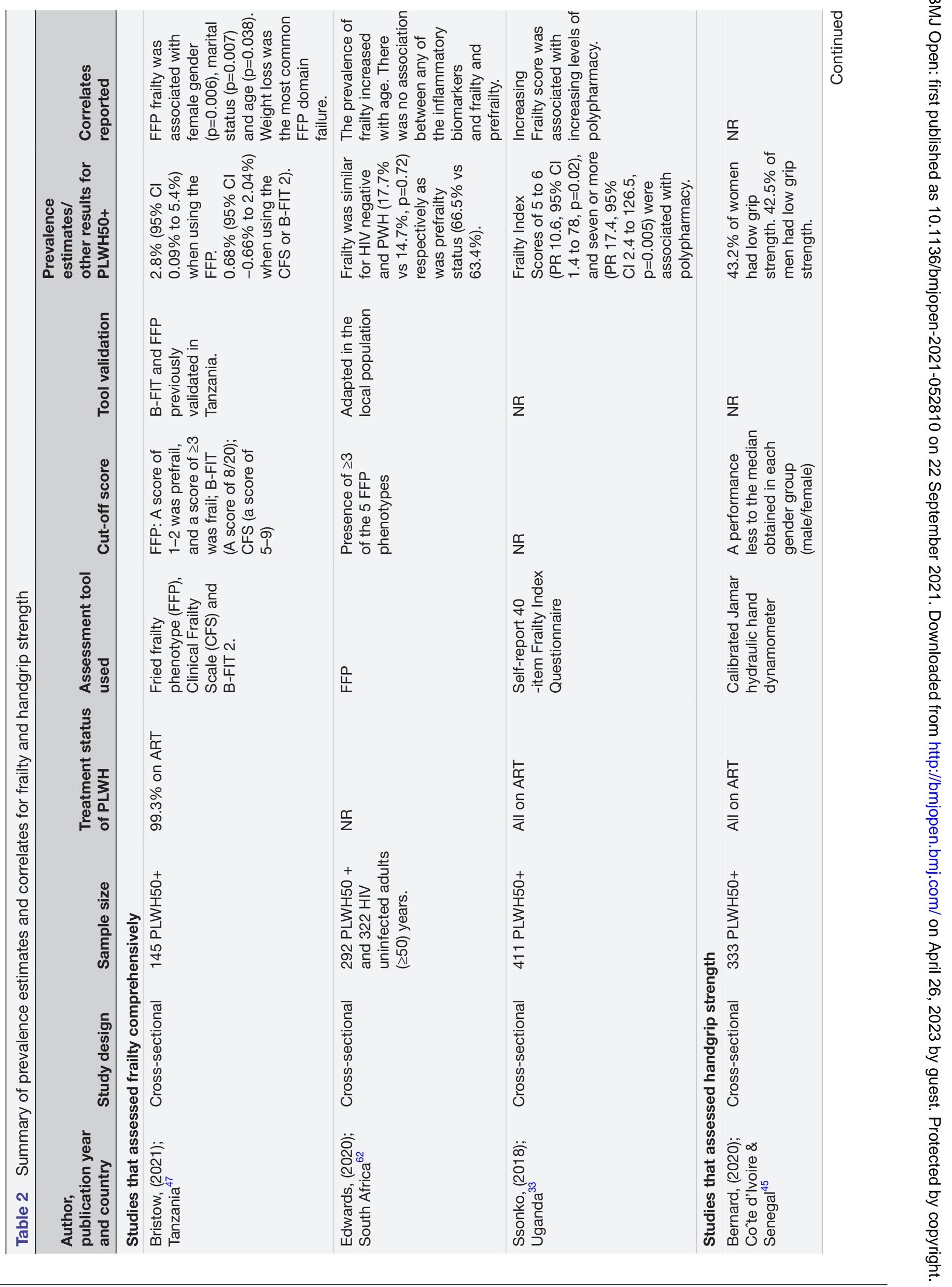




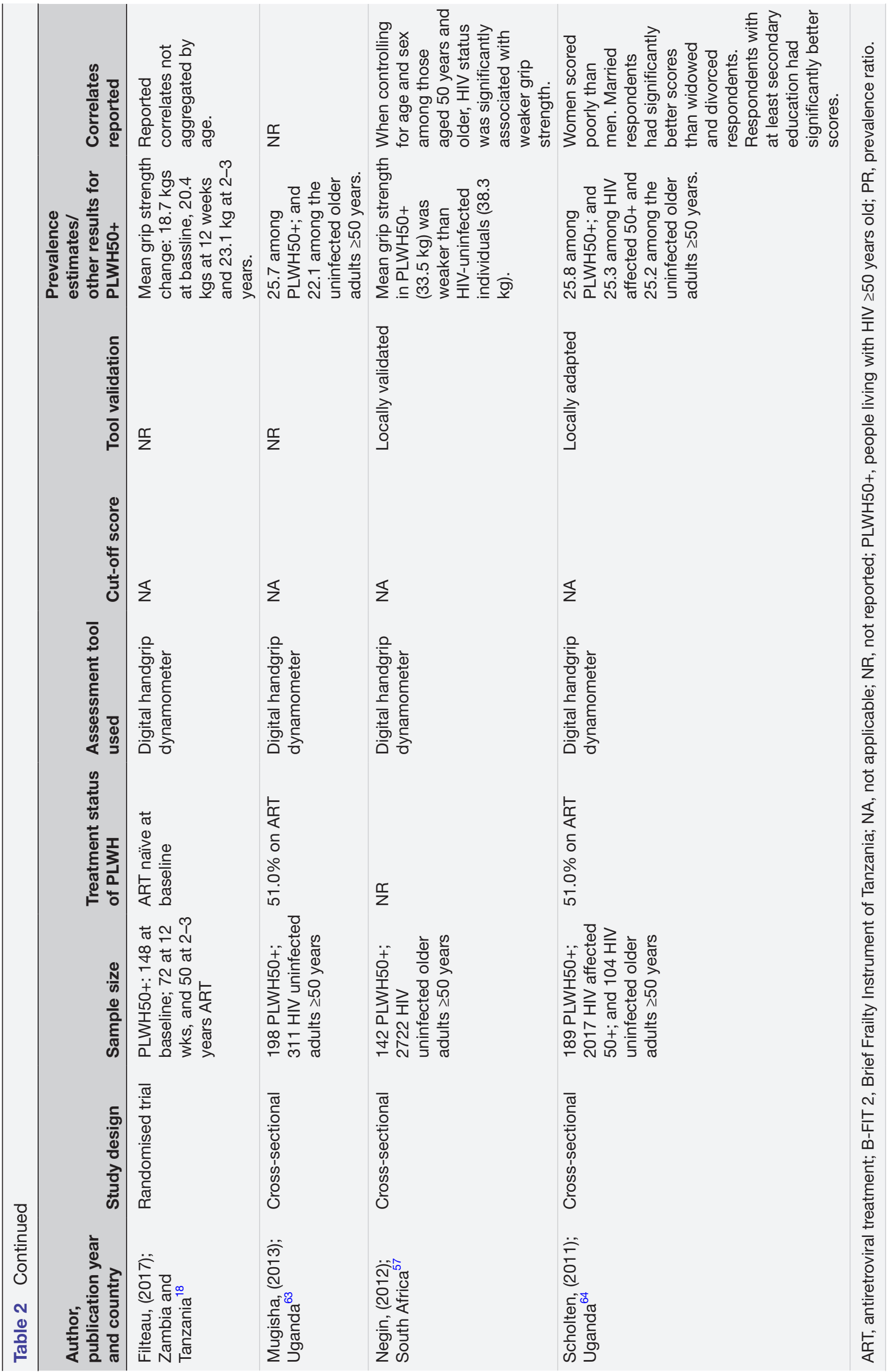




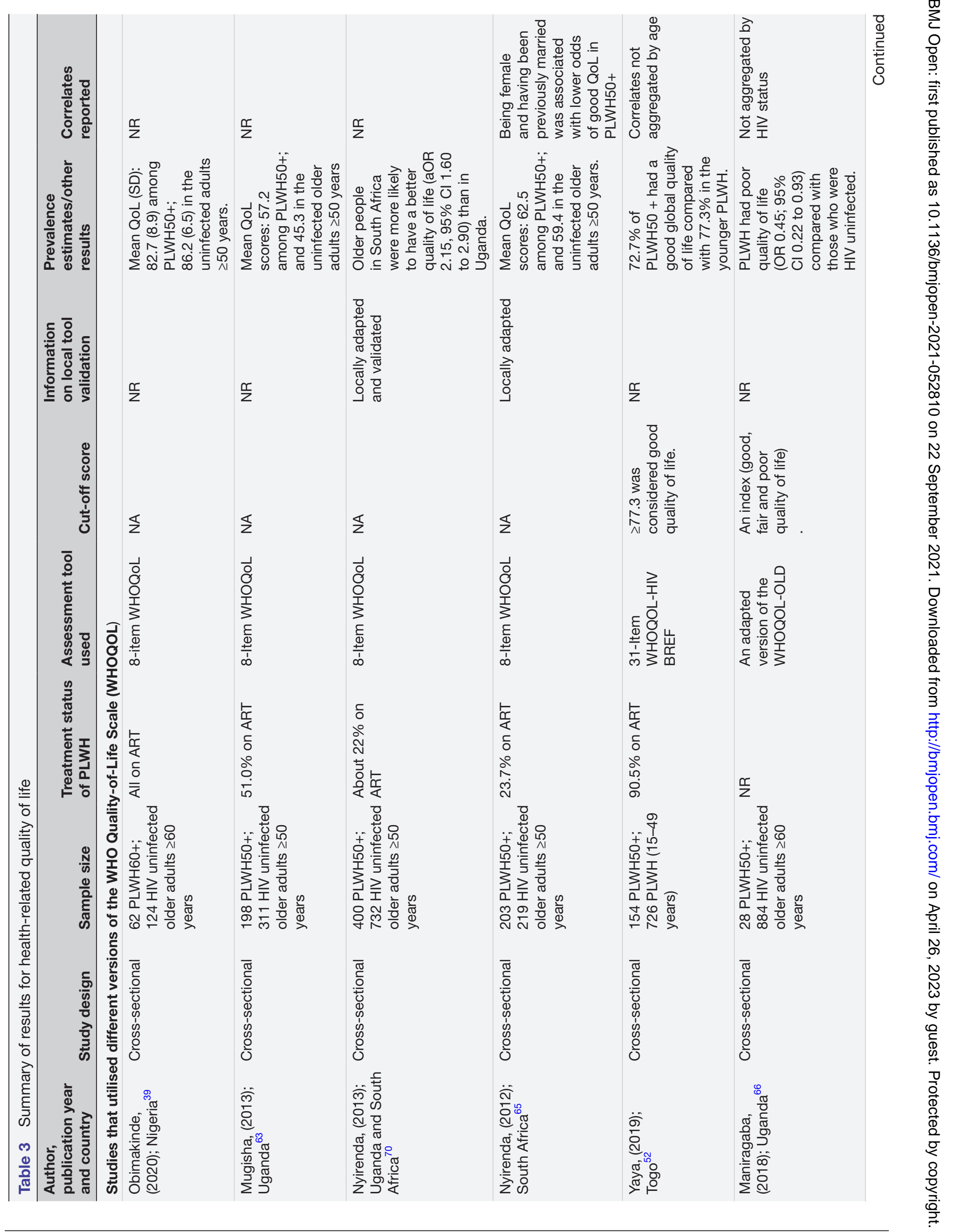




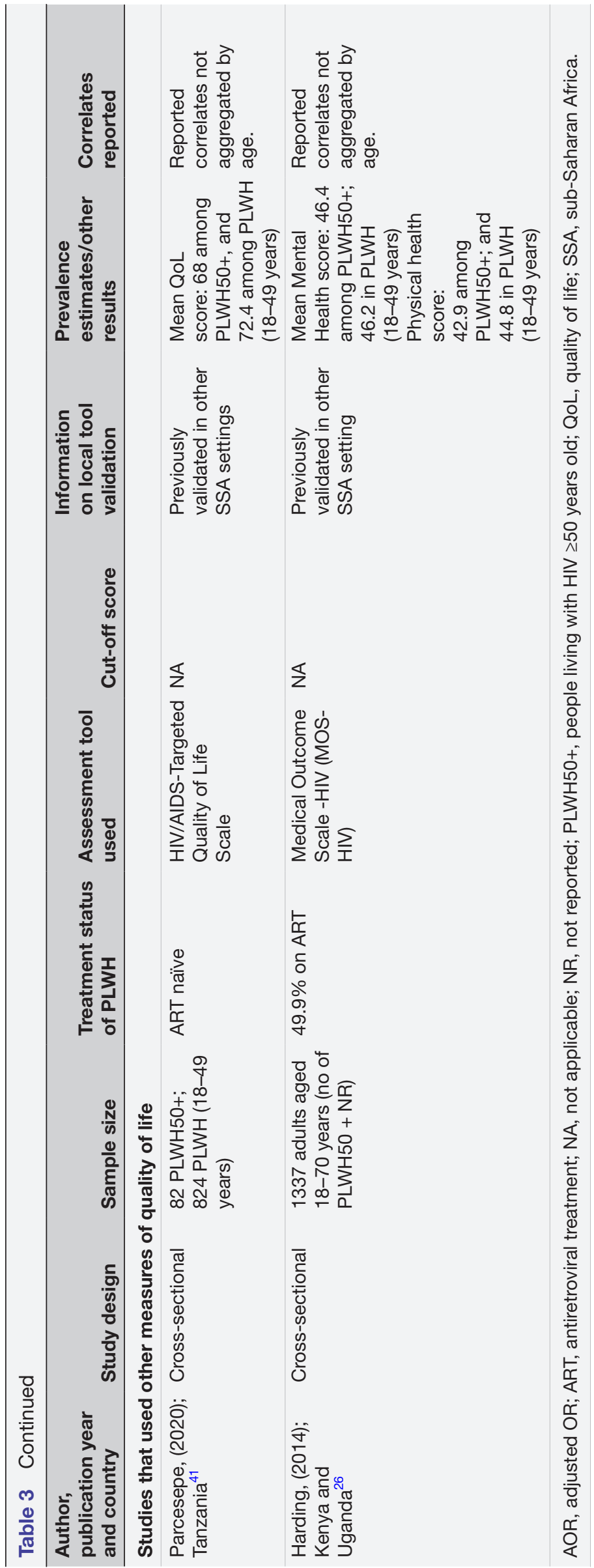




\section{DISCUSSION}

\section{Summary of main findings}

Our review shows that the research on depression, anxiety, cognitive function, frailty and HRQoL among PLWH50 + in SSA is still scanty and largely concentrated in Uganda, South Africa and Ethiopia. Overall, PLWH50 + presented with wide-ranging prevalence of depression (6\% to $59 \%)$, cognitive impairment (4\% to $61 \%)$ and frailty $(3 \%-15 \%)$. Additionally, the correlates of these outcomes were rarely investigated. Nonetheless, those reported were mainly sociodemographic variables, many of which were inconsistent. We find the current evidence inadequate to characterise the real burden and determinants of these outcomes in the region, partly because of the limited number of well-designed studies, heterogeneous findings, and substantial methodological limitations observed in many included studies. Therefore, any policies and programmes about the well-being of PLWH50 + in the region have been made on the basis of minimal evidence. The rising numbers of PLWH50 + in SSA and the potentially elevated burden of age-associated comorbidities in this population warrant more research across the region. Such data will provide the evidence needed to develop appropriate policies, programmes and interventions for the successful ageing of PLWH in SSA.

\section{Depression and anxiety}

Depression was the most frequently reported outcome in about half of the included studies. This finding is consistent with the available literature on HIV, which recognises depression as the most commonly occurring psychiatric complication in this population. ${ }^{67}$ However, only two studies investigated anxiety, a CMD with a similar prevalence to those seen in depression. ${ }^{68}$ The under investigation of anxiety disorders is of particular concern because accumulating research involving PLWH50 + in HICs has documented elevated anxiety levels, as high as $65 \% .^{68}$ More research is needed on this outcome in the region.

Our review also noted wide-ranging prevalences of depression and anxiety. This finding corroborates previous review findings on the prevalence of CMDs in the region, although in younger HIV populations. ${ }^{69}$ The observed variation could reflect significant contextual differences across the SSA region, for example, social environmental milieu, healthcare systems, patterns of the burden of diseases and mental health resources, which are likely to alter the risk profile of these adults. ${ }^{70}$ It could also be attributed to potential differences in study populations, study participants and the use of diverse measurement tools (including different cut-offs for the same measures). Indeed, in the current review, depression was assessed using at least 10 different scoring systems with diverse cut-off points. Besides, the majority of the studies did not provide information on the reliability and validity of the tools they used. Hence, it is difficult to draw firm conclusions regarding the reliability, validity and applicability of many of the measures used to report this outcome. The PHQ-9, for instance, was frequently used to report depressive symptoms among the included studies. However, most of the studies relied on previous validation of the tool, the context of which was not given. A recent review examining the validation of brief CMDs screening tools in low-income and middle-income countries did not support the use of PHQ-9 in these settings. It performed poorly in several clinic populations with lower average education but performed very well in high literate populations. $^{71}$ This is especially an important consideration in SSA, where older adults are more likely to have low literacy levels. There is a need for rigorous cultural adaptation of these instruments to generate accurate epidemiological data that will inform proper intervention work among PLWH50 + in SSA.

\section{Hiv-associated cognitive impairment}

The neurological involvement in HIV disease progression remains problematic, even in well-controlled PLWH, resulting in poor quality of life. HIV associated cognitive impairment is deemed one of the most prevalent comorbidities in the era of ART. Ageing with HIV would appear to impose a synergistic impact, whereby increased comorbidities, such as hypertension, diabetes or depression, likely contribute to and maintain HIV-associated cognitive impairment. In HICs, PLWH50 + have consistently been shown to have high levels of cognitive impairments, as high as $50 \%$. The present review found a wide-ranging prevalence of HIV-associated cognitive impairment $(4 \%-61 \%)$. Our observation is consistent with the available literature, where the prevalence has been noted to vary widely depending on sources used and appears dependent on the populations studied, and approaches used. $^{72}$

Brief cognitive screening tools such as the Mini Mental State Examination and International HIV Dementia Scale (IHDS) were frequently used to document the prevalence of cognitive impairment compared with comprehensive neuropsychological batteries. While the use of the brief screeners in low-resource settings is well understood given the resource gap, researchers should exercise caution on the tools they use. Recent reviews evaluating brief screening tools for neurocognitive impairment in HIV/AIDS have indicated that some of the commonly used tools, for example, the IHDS perform poorly, especially in screening milder cognitive impairments. ${ }^{73} 74$ In the current review, information on tool reliability or validity was rarely reported. One of the studies reported a limited diagnostic accuracy of the IHDS. However, the OCS-Plus, one of the most recent screening tools, is promising. So far, the tool has only been used in South Africa among older adults living with HIV, and its initial evaluation yielded excellent construct and external validity. ${ }^{75}$ This tool has the potential to address some of the unique challenges and gaps facing resource-limited settings in screening for HAND, including difficulty in performing long test batteries, limited screening tools and a shortage of clinical staff. 


\section{Frailty}

Only two studies quantified the prevalence of frailty among PLWH50+, ranging from $3 \%$ to $15 \%$. One of the studies was exclusively conducted among PLWH50+, yielding a low frequency of frailty (3\%). In the other study, conducted in rural South Africa, the prevalence of frailty was similar between PLWH50 + and their uninfected counterparts ( $15 \%$ vs $18 \%$, respectively). This finding supports the growing evidence in SSA, which suggest that older on effective ART have similar or better health profiles than their uninfected counterparts, contrary to what has been observed in HICs. At the global stage, the frailty prevalence in SSA appears relatively lower than that of HICs which ranges from $5 \%$ to $29 \% .^{76}$ The population reaching older age in many SSA settings may be relatively fit/active, still working, which may be true of PLWH, potentially favouring better physical health in these individuals. Nonetheless, given the limited number of studies on this outcome in SSA, these findings remain preliminary and inconclusive and highlight the urgent need for more studies to shed more light on these observations.

\section{Health-related quality of life}

For some time, HRQoL has emerged as a salient indicator of HIV care and an essential target for HIV-related research, aiming to expand the service paradigm continuum. ${ }^{77}$ This new quality of life frontier has been proposed as the 'fourth 90' to the original '90-90-90' testing and treatment target championed by UNAIDS: to ensure that $90 \%$ of people with viral load suppression have good HRQoL. ${ }^{77}$ In the current review, research on HRQoL among PLWH50 + was scanty. Thus, little is known on the risk and protective factors of patientreported HRQoL. Further studies should explore the independent effects of aging-related conditions such as CMDs, cognitive impairments and frailty on HRQoL among PLWH50 + in SSA to support healthy ageing even in the context of a chronic illness such as HIV infection.

Despite the observed limitations, the evidence of better mental health, ${ }^{28445051545859}$ as well as cognition, ${ }^{5361}$ frailty/handgrip strength ${ }^{4762} 63$ and quality of life, ${ }^{65}$ among PLWH50 + on ART (mainly among the robust studies with low risk of bias) compared with uninfected older adults in the current review was striking. This observation could be part of an emerging body of evidence from SSA showing that the mental and wellbeing outcomes among PLWH are comparable to those without HIV. The mechanisms responsible for this observation are not well established and require further examination across many settings in SSA. However, it could be attributed to a few plausible reasons, including (1) the direct impact of ART on viral replication, inflammatory pathways and downstream disease pathophysiology, the so-called 'ART advantage', ${ }^{78}{ }^{79}$ (2) a survivorship bias among PLWH50 + who access and remain in HIV care and (3) beneficial spillover effects of ART programmes such as enhanced access to and utilisation of primary care services resulting in early identification and management of comorbid conditions. ${ }^{79-81}$ This observation could also be related to the fact that PLWH50 + on ART are more likely to use biomedical facilities due to better education and higher socioeconomic status than their HIV uninfected peers. More studies, preferably longitudinal ones, will shed more light on this observation. As it stands, however, this finding offers an interesting contrast to the commonly reported findings from HICs, which generally show that PLWH50 + present with worse physical and mental health outcomes compared with age-matched HIV uninfected people. ${ }^{568} 82$ These findings may suggest context-specific differences in the drivers of physical and mental outcomes, including the demographic and risk factor profile as well as the role of engagement in HIV care-among those with and without HIV, especially when comparing low-income and middle-income to HICs. Future studies should explore the extent to which HIV programmes could be promoting the well-being of PLWH50 + in SSA compared with HIV uninfected older adults in the general population who are likely to bear the brunt of the prevailing poor health systems and primary care systems in the region.

\section{The correlates of mental and well-being outcomes}

The correlates of CMDs, cognitive impairment, frailty and HRQoL have been scarcely investigated in SSA. In the current review, the frequently reported correlates were sociodemographic in nature, many of which (eg, age, formal education levels and wealth status) were inconsistent. However, being female was consistently associated with declining mental health, cognitive function, HRQoL and frailty status. This finding mirrors the results of a recent review of older women's physical and psychological health residing in SSA, which indicated that women were significantly more likely to report poor health compared with men. ${ }^{83}$ This finding highlights the need for adequate representation of both sexes in research studies, incorporating a gender lens in their analyses to understand better the unique health challenges that each gender faces. Further studies are also urgently needed in SSA to identify potential risk and protective factors, especially those that could be modified, to suggest how to best integrate the management of these impairments in HIV care guidelines in SSA. Future studies should explore the influence of HIV-related factors, psychosocial risk (eg, loneliness, ageism, stigma, social support, living arrangements) and other comorbidities on the health and wellbeing of PLWH50+. To promote healthy ageing in the context of chronic conditions, such as HIV infection, the psychological and social domains are especially critical as they could be a way to compensate for physiological limitations (ie, multiple chronic conditions) and allow even in the context of disease and disability, to experience optimal quality of life.

\section{Strengths and limitations}

To our knowledge, this is the first comprehensive review of CMDs, cognitive impairment, frailty and HRQoL 
among PLWH50 + living in SSA. Our expanded search of five online databases and snowballing techniques minimised the risk of missing relevant studies. However, our review should be considered in the context of important limitations. Despite a thorough search strategy, some articles may have been missed, especially in excluding non-English language studies. However, these were very few. The substantial amount of heterogeneity across the studies in terms of, for example, study design, operationalisation of age, definitions of outcomes and measures made comparisons difficult and did not allow a metaanalysis to be done. Furthermore, most of the studies recruited PLWH50 + from HIV clinics whose attendees may represent the less-healthy end of the spectrum of service users leading to overestimation of reported outcomes. Conversely, those at the filter end of the cohort may be more able to attend or be proactive concerning their well-being, leading to underestimation of outcomes. Besides, the substantial methodological limitations observed might have biased the reported findings, hence limiting their external validity.

\section{CONCLUSIONS}

Overall, the existing research on the burden and determinants of mental and well-being outcomes among PLWH50 + in SSA is scantly addressed. We also noted substantial limitations in many of the included studies, which need to be addressed in future studies. Our findings underscore the need to increase the evidence base of these outcomes in the region. Future studies should: (1) explicitly target older persons $\geq 50$ years living with HIV, (2) carefully select a comparison group and have sufficient sample sizes for adequate statistical testing, (3) expand the geographical distribution of these studies in many SSA settings and use adequately standardised survey tools adapted for the region and (4) generate more data on the risk and determinants of these outcomes using a gender lens. There is also an ongoing need for well-designed longitudinal cohort studies to study the temporal patterns of these outcomes in the region.

Acknowledgements The authors would like to thank the Director of Kenya Medical Research Institute for granting permission to publish this work.

Contributors PNM and AAA conceptualised and designed the review. PNM and AM reviewed titles, abstracts and full-text papers for eligibility as well as data extraction. PNM wrote the first draft of the manuscript. AM, CRJCN, RW and AAA reviewed and edited the prepared manuscript.

Funding This work was funded by the Wellcome Trust International Master's Fellowship to PNM (Grant number 208283/Z/17/Z). Further funding supporting this work was from (1) the Medical Research Council (Grant number MR/M025454/1) to AAA. This award is jointly funded by the UK Medical Research Council (MRC) and the UK Department for International Development (DFID) under MRC/DFID concordant agreement and is also part of the EDCTP2 programme supported by the European Union; (2) DELTAS Africa Initiative (DEL-15-003). The DELTAS Africa Initiative is an independent funding scheme of the African Academy of Sciences (AAS)'s Alliance for Accelerating Excellence in Science in Africa (AESA) and supported by the New Partnership for Africa's Development Planning and Coordinating Agency (NEPAD Agency) with funding from the Wellcome Trust (107769/Z/10/Z) and the UK government. For the purpose of Open Access, the author has applied a CC-BY public copyright licence to any accepted manuscript version arising from this submission.

Disclaimer The funders did not have a role in the design and conduct of the study or interpretation of study findings. The views expressed in this publication are those of the author(s) and not necessarily those of AAS, NEPAD Agency, Wellcome Trust or the UK government.

Map disclaimer The inclusion of any map (including the depiction of any boundaries therein), or of any geographic or locational reference, does not imply the expression of any opinion whatsoever on the part of BMJ concerning the legal status of any country, territory, jurisdiction or area or of its authorities. Any such expression remains solely that of the relevant source and is not endorsed by BMJ. Maps are provided without any warranty of any kind, either express or implied.

Competing interests None declared.

Patient consent for publication Not applicable.

Ethics approval This work is part of a PhD project to the first author. The project has obtained ethical clearance from the Kenya Medical Research Institute-Scientific and Ethics Review Unit (KEMRI/SERU/CGMR-C/152/3804).

Provenance and peer review Not commissioned; externally peer reviewed.

Data availability statement All data relevant to the study are included in the article or uploaded as online supplemental information. All data underlying the results are available as part of the article, and no additional source data are required.

Supplemental material This content has been supplied by the author(s). It has not been vetted by BMJ Publishing Group Limited (BMJ) and may not have been peer-reviewed. Any opinions or recommendations discussed are solely those of the author(s) and are not endorsed by BMJ. BMJ disclaims all liability and responsibility arising from any reliance placed on the content. Where the content includes any translated material, BMJ does not warrant the accuracy and reliability of the translations (including but not limited to local regulations, clinical guidelines, terminology, drug names and drug dosages), and is not responsible for any error and/or omissions arising from translation and adaptation or otherwise.

Open access This is an open access article distributed in accordance with the Creative Commons Attribution 4.0 Unported (CC BY 4.0) license, which permits others to copy, redistribute, remix, transform and build upon this work for any purpose, provided the original work is properly cited, a link to the licence is given, and indication of whether changes were made. See: https://creativecommons.org/ licenses/by/4.0/.

ORCID iD

Patrick Nzivo Mwangala http://orcid.org/0000-0001-9046-1465

\section{REFERENCES}

1 United Nations,. World population ageing 2019: Highlights, in ST/ ESA/SER. A/430, 2019.

2 Autenrieth CS, Beck EJ, Stelzle D, et al. Global and regional trends of people living with HIV aged 50 and over: estimates and projections for 2000-2020. PLoS One 2018;13:e0207005.

3 O'Brien KK, Ibáñez-Carrasco F, Solomon P, et al. Research priorities for rehabilitation and aging with HIV: a framework from the CanadaInternational HIV and rehabilitation research collaborative (CIHRRC). AIDS Res Ther 2020;17:21.

4 Sabin CA, Reiss P. Epidemiology of ageing with HIV: what can we learn from cohorts? AIDS 2017;31 Suppl 2:S121-8.

5 Brennan-Ing M, Ramirez-Valles J, Tax A. Aging with HIV: health policy and advocacy priorities. Health Educ Behav 2021;48:5-8.

6 Milic J, Russwurm M, Cerezales Calvino A, et al. European cohorts of older HIV adults: poppy, AGEhIV, GEPPO, cobra and FUNCFRAIL. Eur Geriatr Med 2019;10:247-57.

7 Brothers TD, Rockwood K. Frailty: a new vulnerability indicator in people aging with HIV. Eur Geriatr Med 2019;10:219-26.

8 Gustafson DR, Shi Q, Thurn M, et al. Frailty and Constellations of Factors in Aging HIV-infected and Uninfected Women--The Women's Interagency HIV Study. J Frailty Aging 2016;5:43.

9 Levett T. Frailty prevalence and predictors in older adults with HIV. University of Brighton, 2017.

10 Saka S, Oosthuizen F, Nlooto M. National policies and older people's healthcare in sub-Saharan Africa: a scoping review. Ann Glob Health 2019;85:91

11 Nabalamba A, Chikoko M. Aging population challenges in Africa. African Development Bank, Chief Economist Complex, 2011. 
12 Joint United Nations Programme on HIV/AIDS,. The Gap Report. Geneva, Switzerland: UNAIDS, 2014.

13 World Health Organization. Health statistics and information systems: SAGE HIV studies, 2020. Available: https://www.who.int/healthinfo/ sage/hiv_studies/en/ [Accessed 23 June 2020].

14 Gómez-Ölivé FX, Montana L, Wagner RG, et al. Cohort profile: health and ageing in Africa: a longitudinal study of an indepth community in South Africa (HAALSI). Int J Epidemiol 2018;47:689-90.

15 National Institutes of Health. Ugandan non-communicable diseases and aging cohort (UGANDAC), 2020. Available: https://clinicaltrials. gov/ct2/show/NCT02445079 [Accessed 23 June 2020].

16 Page MJ, Moher D, Bossuyt PM, et al. PRISMA 2020 explanation and elaboration: updated guidance and exemplars for reporting systematic reviews. BMJ 2021;372:n160.

17 Stang A. Critical evaluation of the Newcastle-Ottawa scale for the assessment of the quality of nonrandomized studies in metaanalyses. Eur J Epidemiol 2010;25:603-5.

18 Filteau S, PrayGod G, Woodd SL, et al. Nutritional status is the major factor affecting grip strength of African HIV patients before and during antiretroviral treatment. Trop Med Int Health 2017;22:1302-13.

19 Akena D, Musisi S, Joska J, et al. The association between AIDS related stigma and major depressive disorder among HIV-positive individuals in Uganda. PLoS One 2012;7:e48671.

20 Asangbeh SL, Sobngwi JL, Ekali GL, et al. Predictors of depression among patients on art in a rural health district in North West Cameroon. AIDS Care 2016;28:205-8.

21 Atashili J, Gaynes BN, Pence BW, et al. Prevalence, characteristics and correlates of a positive-dementia screen in patients on antiretroviral therapy in Bamenda, Cameroon: a cross-sectional study. BMC Neurol 2013;13:86.

22 Cassimjee N, Motswai PK. Neuropsychological profiles of adults and older adults with HIV. S Afr J Psychol 2017;47:35-45.

23 Cholera R, Pence BW, Gaynes BN, et al. Depression and engagement in care among newly diagnosed HIV-infected adults in Johannesburg, South Africa. AIDS Behav 2017;21:1632-40.

24 Duko B, Geja E, Zewude M, et al. Prevalence and associated factors of depression among patients with HIV/AIDS in Hawassa, Ethiopia, cross-sectional study. Ann Gen Psychiatry 2018;17:45.

25 Eaton P, Lewis T, Kellett-Wright J, et al. Risk factors for symptomatic HIV-associated neurocognitive disorder in adults aged 50 and over attending a HIV clinic in Tanzania. Int J Geriatr Psychiatry 2020;35:1198-208

26 Harding R, Simms V, Penfold S, et al. Quality of life and wellbeing among HIV outpatients in East Africa: a multicentre observational study. BMC Infect Dis 2014;14:613.

27 Kaharuza FM, Bunnell R, Moss S, et al. Depression and CD4 cell count among persons with HIV infection in Uganda. AIDS Behav 2006;10:105-11.

28 Manne-Goehler J, Kakuhikire B, Abaasabyoona S, et al. Depressive symptoms before and after antiretroviral therapy initiation among older-aged individuals in rural Uganda. AIDS Behav 2019;23:564-71.

29 Musinguzi K, Obuku A, Nakasujja N, et al. Association between major depressive disorder and pro-inflammatory cytokines and acute phase proteins among HIV-1 positive patients in Uganda. BMC Immunol 2018;19:1.

30 Olagunju AT, Adeyemi JD, Erinfolami AR, et al. Factors associated with anxiety disorders among HIV-positive attendees of an HIV clinic in Lagos, Nigeria. Int J STD AIDS 2012;23:389-93.

31 Olley BO, Adebayo KO, Ogunde MJ, et al. Psychosocial factors predicting severity of depression among treatment-seeking HIV/ AIDS patients: a multi-site Nigerian study. Niger J Clin Pract 2017:20:296-302.

32 Shumba C, Atukunda R, Imakit R, et al. Prevalence of depressive symptoms amongst highly active antiretroviral therapy (HAART) patients in AIDSRelief Uganda. J Public Health Afr 2013;4:e19.

33 Ssonko M, Stanaway F, Mayanja HK, et al. Polypharmacy among HIV positive older adults on anti-retroviral therapy attending an urban clinic in Uganda. BMC Geriatr 2018;18:125.

34 Tesfaw G, Ayano G, Awoke T, et al. Prevalence and correlates of depression and anxiety among patients with HIV on-follow up at alert Hospital, Addis Ababa, Ethiopia. BMC Psychiatry 2016;16:368.

35 Tsegaw M, Andargie G, Alem G, et al. Screening HIV-associated neurocognitive disorders (hand) among HIV positive patients attending antiretroviral therapy in South Wollo, Ethiopia. J Psychiatr Res 2017;85:37-41.

36 Berhe H, Bayray A. Prevalence of depression and associated factors among people living with HIV/AIDS in tigray, North Ethiopia: a cross sectional hospital based study. International Journal of Pharmaceutical Sciences and Research 2013;4:765.

37 Eshetu DA, Woldeyohannes SM, Kebede MA. Prevalence of depression and associated factors among HIV/AIDS patients attending art clinic at Debrebirhan referral Hospital, North Showa, Amhara region, Ethiopia. Clin Psychiatry 2015;1:1-7.

38 Mugendi AG, Kubo MN, Nyamu DG, et al. Prevalence and correlates of neurocognitive disorders among HIV patients on antiretroviral therapy at a Kenyan Hospital. Neurol Res Int 2019;2019:1-10.

39 Obimakinde AM, Adebusoye L, Achenbach C, et al. Going beyond giving antiretroviral therapy: multimorbidity in older people aging with HIV in Nigeria. AIDS Res Hum Retroviruses 2020;36:180-5.

40 Oumar G, Yacine Z, Herve T, et al. Neurocognitive disorders in people living with human immunodeficiency virus aged 50 years old and more in Yalgado Ouédraogo teaching hospital. American Journal of Internal Medicine 2020;8:177-81.

41 Parcesepe AM, Nash D, Tymejczyk O, et al. Gender, HIV-related stigma, and health-related quality of life among adults enrolling in HIV care in Tanzania. AIDS Behav 2020;24:142-50.

42 Torgersen J, Bellamy SL, Ratshaa B, et al. Impact of efavirenz metabolism on loss to care in older HIV+ Africans. Eur J Drug Metab Pharmacokinet 2019;44:179-87.

43 Abadiga M. Depression and its associated factors among HIV/ AIDS patients attending art clinics at Gimbi General Hospital, West Ethiopia, 2018. BMC Res Notes 2019;12:527.

44 Asrat B, Lund C, Ambaw F, et al. Major depressive disorder and its association with adherence to antiretroviral therapy and quality of life: cross-sectional survey of people living with HIV/AIDS in Northwest Ethiopia. BMC Psychiatry 2020;20:462.

45 Bernard C, Font H, Diallo Z, et al. Prevalence and factors associated with physical function limitation in older West African people living with HIV. PLoS One 2020;15:e0240906.

46 Bernard C, Font H, Diallo Z, et al. Prevalence and factors associated with severe depressive symptoms in older West African people living with HIV. BMC Psychiatry 2020;20:442.

47 Bristow C, George G, Hillsmith G, et al. Low levels of frailty in HIVpositive older adults on antiretroviral therapy in northern Tanzania. $J$ Neurovirol 2021;27:58-69.

48 Kalomo EN, Jun JS, Lee K, et al. Hiv stigma, resilience and depressive symptoms among older adults living with HIV in rural Namibia. Afr J AIDS Res 2020;19:198-205.

49 Kellett-Wright J, Flatt A, Eaton P, et al. Screening for HIV-associated neurocognitive disorder (hand) in adults aged 50 and over attending a government HIV clinic in Kilimanjaro, Tanzania. Comparison of the International HIV dementia scale (IHDS) and idea six item dementia screen. AIDS Behav 2021;25:542-53.

50 Motumma A, Negesa L, Hunduma G, et al. Prevalence and associated factors of common mental disorders among adult patients attending HIV follow up service in Harar town, eastern Ethiopia: a cross-sectional study. BMC Psychol 2019;7:11.

51 Moucheraud C, Paul-Schultz J, Mphande M, et al. A multidimensional characterization of aging and wellbeing among HIVpositive adults in Malawi. AIDS Behav 2021;25:571-81.

52 Yaya I, Djalogue L, Patassi AA, et al. Health-Related quality of life among people living with HIV/AIDS in Togo: individuals and contextual effects. BMC Res Notes 2019;12:140.

53 Kobayashi LC, Mateen FJ, Montana L, et al. Cognitive Function and Impairment in Older, Rural South African Adults: Evidence from "Health and Aging in Africa: A Longitudinal Study of an INDEPTH Community in Rural South Africa". Neuroepidemiology 2019;52:32-40.

54 Geldsetzer P, Vaikath M, Wagner R, et al. Depressive symptoms and their relation to age and chronic diseases among middle-aged and older adults in rural South Africa. J Gerontol A Biol Sci Med Sci 2019;74:957-63.

55 Kinyanda E, Kuteesa M, Scholten F, et al. Risk of major depressive disorder among older persons living in HIV-endemic central and southwestern Uganda. AIDS Care 2016;28:1516-21.

56 Mugisha JO, Schatz EJ, Randell M, et al. Chronic disease, risk factors and disability in adults aged 50 and above living with and without HIV: findings from the wellbeing of older people study in Uganda. Glob Health Action 2016;9:31098.

57 Negin J, Martiniuk A, Cumming RG, et al. Prevalence of HIV and chronic comorbidities among older adults. AIDS 2012;26 Suppl 1:S55-63.

58 Nyirenda M, Chatterji S, Rochat T, et al. Prevalence and correlates of depression among HIV-infected and -affected older people in rural South Africa. J Affect Disord 2013;151:31-8.

59 Rohr JK, Manne-Goehler J, Gómez-Olivé FX, et al. Hiv treatment cascade for older adults in rural South Africa. Sex Transm Infect 2020;96:271-6.

60 Joska JA, Dreyer AJ, Nightingale S, et al. Prevalence of HIV1 infection in an elderly rural population and associations with neurocognitive impairment. AIDS 2019;33:1765-71. 
61 Asiimwe SB, Farrell M, Kobayashi LC, et al. Cognitive differences associated with HIV serostatus and antiretroviral therapy use in a population-based sample of older adults in South Africa. Sci Rep 2020;10:16625.

62 Edwards A, Siedner MJ, Nash S, et al. HIV serostatus, inflammatory biomarkers and the frailty phenotype among older people in rural KwaZulu-Natal, South Africa. Afr J AIDS Res 2020;19:177-85.

63 Mugisha J, Scholten F, Owilla S, et al. Caregiving responsibilities and burden among older people by HIV status and other determinants in Uganda. AIDS Care 2013;25:1341-8.

64 Scholten F, Mugisha J, Seeley J, et al. Health and functional status among older people with HIV/AIDS in Uganda. BMC Public Health 2011;11:886

65 Nyirenda M, Chatterji S, Falkingham J, et al. An investigation of factors associated with the health and well-being of HIV-infected or HIV-affected older people in rural South Africa. BMC Public Health 2012;12:259.

66 Maniragaba F, Kwagala B, Bizimungu E, et al. Predictors of quality of life of older persons in rural Uganda: A cross sectional study. AAS Open Res 2018;1:22.

67 Nanni MG, Caruso R, Mitchell AJ, et al. Depression in HIV infected patients: a review. Curr Psychiatry Rep 2015;17:530.

68 Brennan-Ing M. Emerging issues in HIV and aging, 2020.

69 Too EK, Abubakar A, Nasambu C, et al. Prevalence and factors associated with common mental disorders in young people living with HIV in sub-Saharan Africa: a systematic review. J Int AIDS Soc 2021;24 Suppl 2:e25705.

70 Nyirenda M, Newell M-L, Mugisha J, et al. Health, wellbeing, and disability among older people infected or affected by HIV in Uganda and South Africa. Glob Health Action 2013;6:19201.

71 Ali G-C, Ryan G, De Silva MJ. Validated screening tools for common mental disorders in low and middle income countries: a systematic review. PLoS One 2016;11:e0156939.

72 Alford K, Vera JH. Cognitive impairment in people living with HIV in the art era: a review. Br Med Bull 2018;127:55-68.

73 Zipursky AR, Gogolishvili D, Rueda S, et al. Evaluation of brief screening tools for neurocognitive impairment in HIV/AIDS: a systematic review of the literature. AIDS 2013;27:2385.
74 Mwangala PN, Newton CR, Abas M, et al. Screening tools for HIVassociated neurocognitive disorders among adults living with HIV in sub-Saharan Africa: a scoping review. AAS Open Res 2018;1:28.

75 Humphreys GW, Duta MD, Montana L, et al. Cognitive function in low-income and low-literacy settings: validation of the tabletbased Oxford cognitive screen in the health and aging in Africa: a longitudinal study of an indepth community in South Africa (HAALSI). J Gerontol B Psychol Sci Soc Sci 2017;72:38-50.

76 Levett TJ, Cresswell FV, Malik MA, et al. Systematic review of prevalence and predictors of frailty in individuals with human immunodeficiency virus. J Am Geriatr Soc 2016;64:1006-14.

77 Lazarus JV, Safreed-Harmon K, Barton SE, et al. Beyond viral suppression of HIV - the new quality of life frontier. BMC Med 2016;14:94.

78 Norcini Pala A, Steca P, Bagrodia R, et al. Subtypes of depressive symptoms and inflammatory biomarkers: an exploratory study on a sample of HIV-positive patients. Brain Behav Immun 2016;56:105-13.

79 Manne-Goehler J, Montana L, Gómez-Olivé FX, et al. The art advantage: health care utilization for diabetes and hypertension in rural South Africa. J Acquir Immune Defic Syndr 2017;75:561-7.

80 Feinstein MJ, Kim J-H, Bibangambah P, et al. Ideal cardiovascular health and carotid atherosclerosis in a mixed cohort of HIVinfected and uninfected Ugandans. AIDS Res Hum Retroviruses 2017;33:49-56.

81 Wouters E, Heunis C, van Rensburg D, et al. Physical and emotional health outcomes after 12 months of public-sector antiretroviral treatment in the free state Province of South Africa: a longitudinal study using structural equation modelling. BMC Public Health 2009;9:103.

82 Langebeek N, Kooij KW, Wit FW, et al. Impact of comorbidity and ageing on health-related quality of life in HIV-positive and HIVnegative individuals. AIDS 2017;31:1471-81.

83 Mangipudi S, Cosco T, Harper S. A systematic review of physical and psychological health and wellbeing of older women in sub-Saharan Africa. J Public Health 2020;42:294-303. 